\title{
A Novel Grouping Mechanism for DSMEMAC Mode
}

\author{
K Suresh, M.Ramesh Patnaik
}

\begin{abstract}
IEEE 802.15.4e introduces deterministic and Synchronous multi-channel extension (DSME) MAC mode to provide deterministic performance. To do so, the super frame structure is divided into multiple contention access slots and contention free slots. However, the standard does not specify the number of devices to access each DSME slots. In this article, we exploit fuzzy logic to group the devices based on the nature of traffic and vary the duration of DSME slot according to the traffic requirements of the devices. Further, we analytically evaluate the collision probability, throughput, energy consumption and delay of DSME mechanism. Results show that the optimal amount of DSME slots associated with fuzzy logic significantly improves efficiency and reduces energy usage.. Finally, extensive simulations are conducted using ns-3 to validate the analytical results.
\end{abstract}

Keywords:IEEE 802.15.4e, DSME, fuzzy logic.

\section{INTRODUCTION}

$\mathrm{W}$ th the increase in low cost and low power wireless sensor devices, green communication has revolutionized the Internet of Things (IoT)[1]. To enhance the networking capability of IoT, IEEE 802.15.4 is introduced [3]. Few of the standards recognized by IEEE 802.15.4 are ZigBee, IETF 6LoWPAN, Wireless HART and ISA100.11a because of their low power consumption [2,4]. It operates in $2.4 \mathrm{GHz}$ of ISM bands. In industrial application like food processing, wireless controlled area networks, transfer of large amount of data with less energy consumption shows a significant impact on the performance of the network. As the available channel time in IEEE 802.15.4 is common to all the devices, there is a performance degradation due to severe contention. To overcome this problem IEEE 802.15 task group introduces IEEE 802.15.4e as an amendment to IEEE 802.15.4 [5]. The new standard introduces five different MAC modes, among which Deterministic and Synchronous multi-channel Extension (DSME) is used to provide deterministic time slots[8]. DSME uses multi-channel to improve aggregate utility of the network. In [6], authors presented a detailed

Revised Manuscript Received on April 27, 2020.

* Correspondence Author

K Suresh*, Research Scholar,Department of Instrument Technology, Andhra University, VSP, India.Email: sureshvarma6277@gmail.com

M. Ramesh Patnaik, Associate Professor ,Department of Instrument Technology, Andhra University, VSP, India. Email: ramesh_patnaik@yahoo.com

(C) The Authors. Published by Blue Eyes Intelligence Engineering and Sciences Publication (BEIESP). This is an open access article under the CC BY-NC-ND license (http://creativecommons.org/licenses/by-nc-nd/4.0/) survey on the performance evaluation of IEEE 802.15.4e network. Authors in [7] dynamically tune the duration of the DSME slots. Among [10-14], several have been proposed to assess the performance of the IEEE 802.15.4e MAC mode.

Each system in the network has specific propagation specifications on an IoT network. Of example, a remote monitoring device that measures the patient's condition has specific sample levels and channel access specifications for a warm ground surface or a pulse detector. All nodes can not therefore be allocated an equivalent-duration DSME location. The length of DSME slots should therefore be adjusted to the propagation criteria of the systems instead of supplying each with an identical DSME slot.

The following article is used to associate systems with identical communication criteria and allocates each category to a DSME slot the length of which differs as per device propagation conditions, using a fuzzy c-means (FCM) clustering algorithm. The DSME conceptual model's performance, energy usage and latency are studied with a basic but reliable research method.

The article is as follows : Section II recommended the maximum slot distribution scheme based on the low. Section III provides a description of the likelihood of collision, efficiency, consumption of energy and latency. The findings and clarification are discussed in Section IV. The summary of this article is given in Section V:

- An empirical model for determining the likelihood of collision, latency, energy usage, and network delay in a dense IoT scenario is created.

- $\quad$ The fuzzy $\mathrm{C}$ mean cluster is used to align the equipment on traffic criteria

- The experimental results show significant network performance gain with the optimum number of DSME spaces.

- $\quad$ The theoretical findings are finally verified using ns-3 simulations.

\section{FCMMETHOD}

The Fuzzy Logic Systems (FLS) in this segment utilizes Fuzzy C-means (FCM) clusters to cluster the equipment with identical communication specifications and to assign each category a DSME slot with a length that differs according to system communication criteria.

Initially growing network unit listens to the frequently transmitted beacons and undergoes affiliation procedures with the AP. Growing device uses its wireless capabilities to approximate the period the channel requires to be reached, before the connection.

Published By:

Blue Eyes Intelligence Engineering DOI: 10.35940/ijeat.C5104.049420

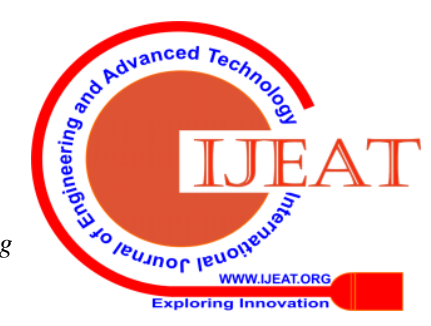




$$
\Delta_{n}=\sum_{i=0}^{R} W_{i} \sigma+E\left[T_{s, n}\right]+\Delta_{f, n} .
$$

Eq's first phrase. (1) is the back-off counter length. $E\left[T_{s, n}\right]$ is the time between the transfer of the packet until

the acknowledgment mechanism is received. $\Delta_{f, n}$ is the length of time the monitor reverse stops owing to secretly recording of several other broadcasts. Hence,

$$
\Delta_{f, n}=R\left(E\left[T_{s, n}\right]+S I F S+T_{\text {ACK_timeout }}\right) \text {. }
$$

\section{ANALYSIS}

We believe that the infrastructure is rotationally symmetric and loaded. We find an interception-free channel and test network uplink efficiency. The channel is split into time slots $\sigma$.We find a network of scale $g$ where all users use the existing DCF system to compete for channel control[ 2]. Before starting the clock, the source is perceived for DIFS time. The backstage is picked from[ 0 , W0-1], where W0 is the limited view window. For each delivery of packets W0 is set to zero and for conflict the cumulative discrepancy span (CWmax) is doubled[9]. A back-off statement is made by,

$$
W_{i}= \begin{cases}2^{i} \times W_{0} ; & 0 \leq i \leq m-1, \\ 2^{m} \times W_{0} ; & m \leq i \leq R,\end{cases}
$$

The cumulative retries are where $m$ is the average W0 and $R$. When the W0 is small, a device gets the transmitting chance. The system begins the back-off counter for two simultaneous transmissions, preceded by the DIFS length. According to the model, the transmission is given by,

$$
\tau_{j}=\sum_{i=0}^{m} b_{i, 0}=\sum_{i=0}^{m} p_{c, j}^{i} b_{0,0} .
$$

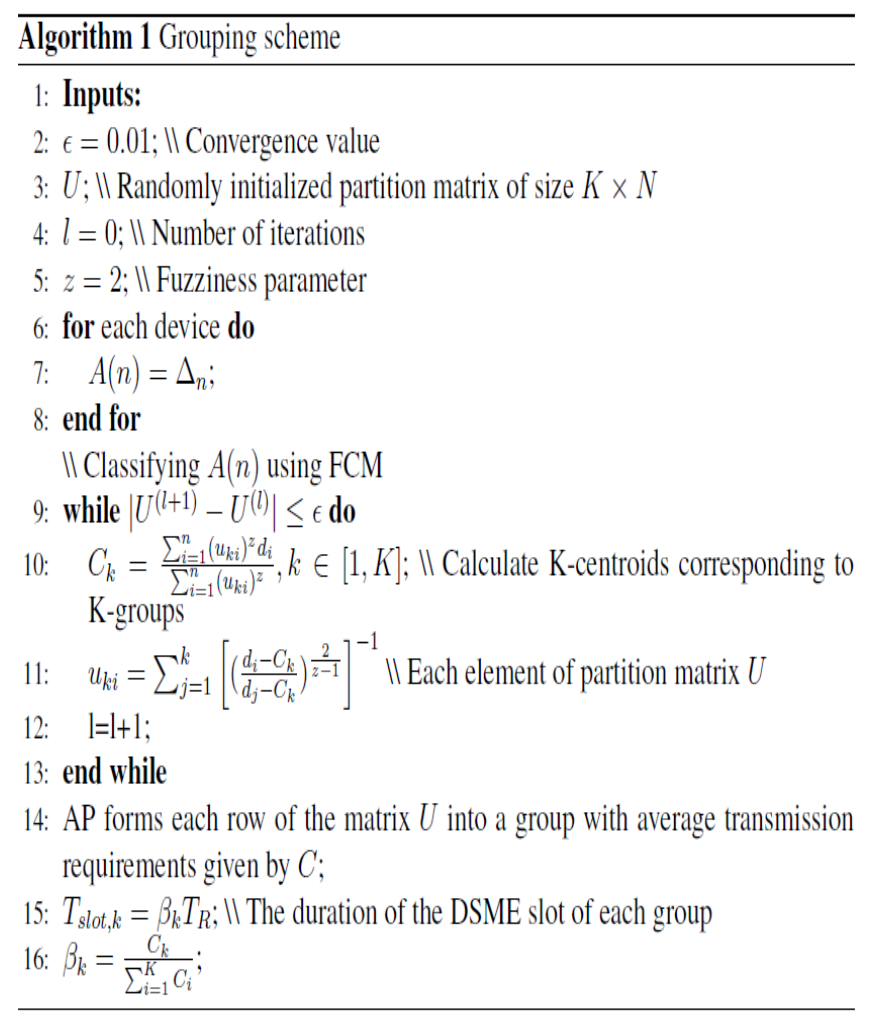

Then the likelihood of a predetermined collision is given,

$$
p_{c, j}=1-\left(1-\tau_{j}\right)^{g-1} \text {. }
$$

Let $P_{t r, j}$ is transmission probability in a $j^{\text {th }}$ slot,

$$
P_{t r, j}=1-\left(1-\tau_{j}\right)^{g}
$$

is an effective packet communication in,

$$
P_{s, j}=\frac{g \tau_{j}\left(1-\tau_{j}\right)^{g-1}}{1-\left(1-\tau_{j}\right)^{g}} .
$$

\section{A. Throughput}

The saturation throughput $S_{j}$ is,

$$
\begin{aligned}
& S_{j}=\frac{\left(\begin{array}{c}
\text { Averageinformation } \\
\text { transmittedinamini-slot }
\end{array}\right)}{\text { Averagedurationofamini-slot }}, \\
& =\frac{P_{t r, j} P_{s, j} E[P]}{\left(1-P_{t r, j}\right) \sigma+P_{t r, j} P_{s, j} T_{s}+P_{t r, j}\left(1-P_{s, j}\right) T_{c}}
\end{aligned}
$$

And the transmission rate of payload and packet frames are calculated as,

$$
\begin{gathered}
T_{E[P]}(\text { Rate })=\frac{8 \times(E[P]+M A C)}{\frac{\text { Rate }}{\text { basic_datarate }} \times L_{\text {sym }}^{\text {basic_datarate }}} \times T_{\text {sym }}+T_{P H Y}, \\
T_{\text {control }}=\frac{8 \times \text { ControlFrame }_{\text {tratratate }}^{\text {basic_datarate }} \times T_{\text {sym }}+T_{P H Y} \cdot}{L_{\text {sym }}}
\end{gathered}
$$

\section{B. Energy consumption}

The energy consumption has four parts:

- $E_{b}$ The resources expended during the retroactive phase.

- $E_{f}$ It is the energy absorbed by freezing the rear counter of a system.

- $E_{s}$ and $E_{c}$ Energies are absorbed by efficient distribution and conflict.

Therefore, the energy consumption is defined as:

$$
\eta_{j}=\frac{E_{b}+E_{f}+E_{s}+E_{c}}{P_{t r, j} P_{s, j} E[P]} .
$$

The respective energies are given by,

$$
\begin{gathered}
E_{b}=E_{f}=E[B] \sigma P_{\text {idle }}, \\
E_{s}=P_{T x}\left(T_{P S \_P o l l}+T_{E[P]}\right)+P_{R x}\left(T_{s}-T_{P S \_P o l l}+T_{E[P]}\right) \\
E_{c}=N_{t}\left[P_{T X}\left(T_{P S \_P o l l}\right)+P_{R x}\left(T_{c}-T_{P S_{-} \text {Poll }}\right)\right]
\end{gathered}
$$

\section{Delay}

The average delay $\left(D_{j}\right)$ is time taken for a successful transmission. Therefore,

$$
D_{j}=\mathbb{E}[B] \sigma+N_{t}\left(T_{c}-T_{A C K}\right)+T_{c} .
$$

\section{Delay}

The average delay $\left(D_{j}\right)$ is time taken for a successful transmission. Therefore,

$$
D_{j}=\mathbb{E}[B] \sigma+N_{t}\left(T_{c}-T_{A C K}\right.
$$




\section{PERFORMANCE EVALUATION}

This section discusses theoretical and emulation results. In Section II, the theoretical framework is tested by MATLAB. The outcomes of the experiment are verified by the accessible software system emulator ns-3.

In this paper, a network of size $g$ is uniformly used around the AP. The AP operates the Fuzzy deduction mechanism to determine the correct number of DSME (K) slots. We take into consideration a network size of $g=256$ machines in which $\mathrm{K}=32$ is the maximum number of DSME slots.

Illustration 1, Fig.2 and Fig. 3 indicates the DSME mechanism's efficiency. We find a channel size divided into classes $K=\{8,16,32,64,128\}$. The graph shows that the efficiency of the DSME system improves with a rise in the amount of classes in terms of performance and power consumption. Since an improvement in $\mathrm{K}$ reduces the community size and the conflict between them. In the meantime, it is found that the energy consumption, delays and a steady decline in production rises marginally for greater $\mathrm{K}$ values. The primary cause for this is the loss of channel resources. While the dispute is reduced for greater $\mathrm{K}$ values, the systems of their respective groups use traditional DCF to reach the channel and wast network period in the back-off process. This negative pattern increasing the saturation limit. Yet the system is inactive and uses fewer power throughout the back-up time. Therefore, energy use is not substantially increased.

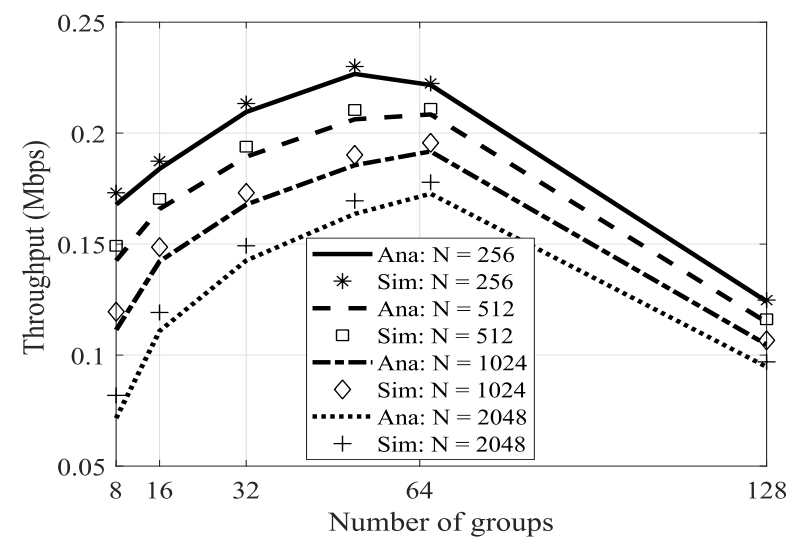

Fig 1. Throughput Vs. network size

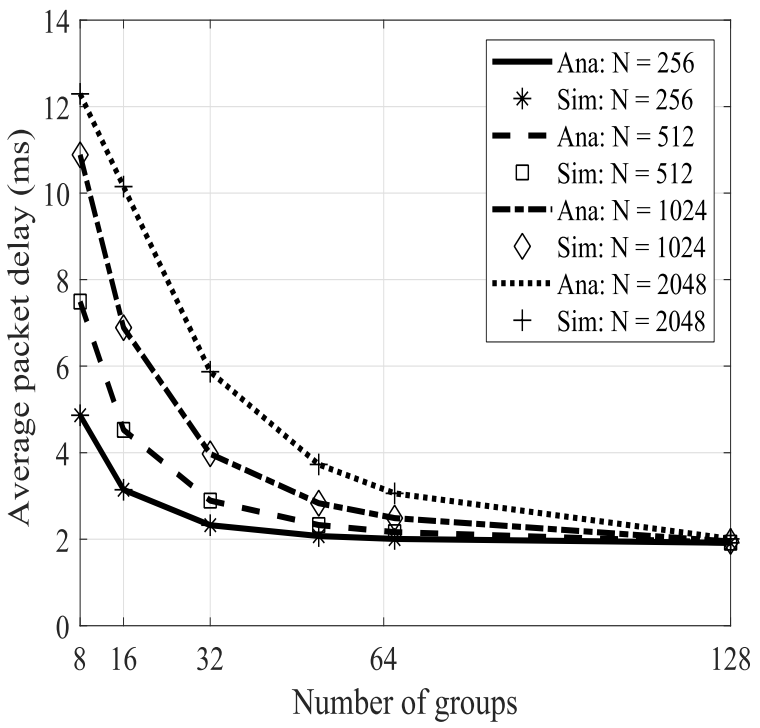

Fig. 2 Delay Vs. network size

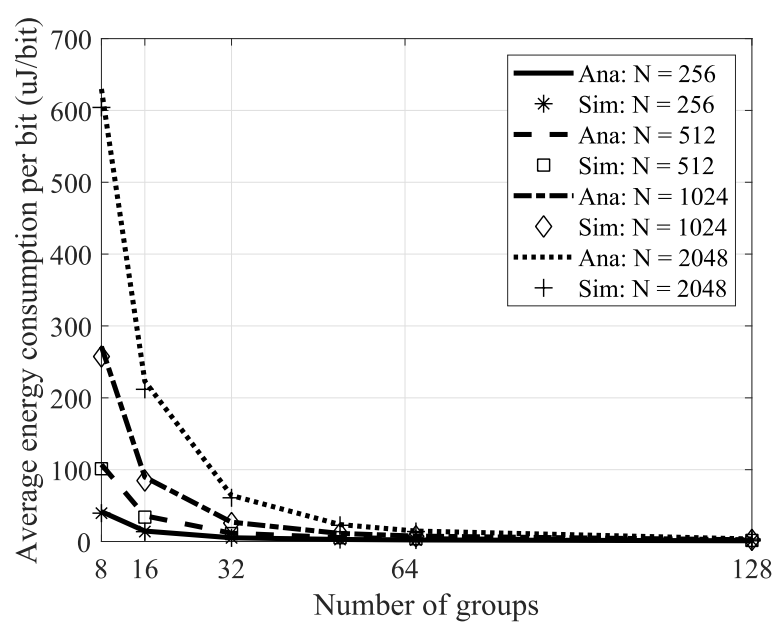

Fig. 3 Energy consumption Vs. network size

Table 2 indicates the difference in the volume of information transmitted in keeping has system communication requirements. We notice a size 32 system separated into $\mathrm{K}\{$ opt $\}=4$. Class 1 with the highest total communication specifications and class 4 for the lowest transmission demands. The length of each DSME slot is specified in Table 2 and is dependent on Algorithm 1. Group1 tools with longer DSME slots enter the channel for a long period and are able to transmit more data than group 4 devices with shorter DSME slots. Statistics from Table 2 reveal that large volumes of data are transmitted by category 1 , with the largest transmission levels relative to lower transmission demand groups. The grouping scheme will also conveniently follow the traffic specifications of various devices.

Table 2 Data in a DSME slot

\begin{tabular}{|c|c|c|c|c|}
\hline \multirow[t]{2}{*}{ Groups } & \multirow[t]{2}{*}{ beta } & \multirow{2}{*}{$\begin{array}{l}\text { Duration of } \\
\text { DSME slot }\end{array}$} & \multicolumn{2}{|c|}{ Grouping scheme } \\
\hline & & & Analytical & Simulation \\
\hline group & 0.3308 & 0.1654 & 229.8801 & 229.8319 \\
\hline group-2 & 0.2951 & 0.1475 & 205.0714 & 205.0284 \\
\hline group-3 & 0.2281 & 0.114 & 158.5116 & 158.4784 \\
\hline group-4 & 0.146 & 0.073 & 101.4586 & 101.4373 \\
\hline \multicolumn{3}{|c|}{ Aggregate throughput } & 694.9216 & 694.776 \\
\hline
\end{tabular}

\section{CONCLUSION}

A FIS grouping scheme for DSME MAC mode is defined in this article. The suggested program uses fugitive c-means to organize the systems in compliance with traffic requirements. A basic mathematical model was introduced in order to determine the likelihood of collision, throughput, energy usage and the latency of the IEEE 802.15.4e DSME. It is evident from the findings that the proposed scheme strengthens the DSME mechanism. Finally, extensive simulation studies were carried out to validate the study findings.

\section{REFERENCES}

1. J. Xu, Y. Andrepoulos, Y. Xiao and M. Schaar, "Non-Stationary Resource Allocation Policies for Delay-Constrained Video Streaming: Application to Video over Internet-of-Things-Enabled Networks," IEEE Journal on Selected Areas in Communications, vol. 32, pp. 782-794, 42014

Published By:

Blue Eyes Intelligence Engineering

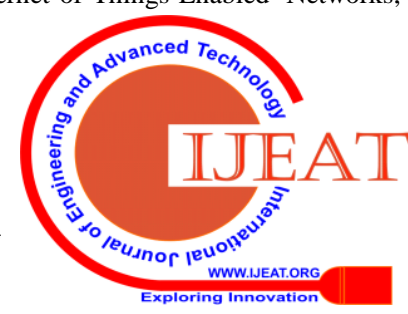


2. B. Velusamy and S. C. Pushpan, "An Enhanced Channel Access Method to Mitigate the Effect of Interference Among Body Sensor Networks for Smart Healthcare," IEEE Sensors Journal, vol. 19, pp. 7082-7088, 82019.

3. G. Patti and L. L. Bello, "A Priority-Aware Multichannel Adaptive Framework for the IEEE 802.15.4e-LLDN," IEEE Transactions on Industrial Electronics, vol. 63, pp. 6360-6370, 102016.

4. M. R. Palattella, N. Accettura, X. Vilajosana, T. Watteyne, L. A. Grieco, G. Boggia and M. Dohler, "Standardized Protocol Stack for the Internet of (Important) Things," IEEE Communications Surveys Tutorials, vol. 15, pp. 1389-1406, 2013.

5. K. Muraoka, T. Watteyne, N. Accettura, X. Vilajosana and K. S. J. Pister, "Simple Distributed Scheduling With Collision Detection in TSCH Networks," IEEE Sensors Journal, vol. 16, pp. 5848-5849, 8 2016.

6. H. Kurunathan, R. Severino, A. Koubaa and E. Tovar, "IEEE 802.15.4e in a Nutshell: Survey and Performance Evaluation," IEEE Communications Surveys Tutorials, vol. 20, pp. 1989-2010, 2018.

7. H. Kurunathan, R. Severino, A. Koubaa and E. Tovar, "DynaMO-Dynamic Multisuperframe Tuning for Adaptive IEEE 802.15.4e DSME Networks," IEEE Access, vol. 7, pp. 122522-122535, 2019.

8. H. Kapil and C. S. R. Murthy, "A Pragmatic Relay Placement Approach in 3-D Space and Q-Learning-Based Transmission Scheme for Reliable Factory Automation Applications," IEEE Systems Journal, vol. 12, pp. 823-833, 32018.

9. D. D. Guglielmo, B. A. Nahas, S. Duquennoy, T. Voigt and G. Anastasi, "Analysis and Experimental Evaluation of IEEE 802.15.4e TSCH CSMA-CA Algorithm," IEEE Transactions on Vehicular Technology, vol. 66, pp. 1573-1588, 22017.

10. F. Chiti, R. Fantacci and A. Tani, "Performance Evaluation of an Adaptive Channel Allocation Technique for Cognitive Wireless Sensor Networks," IEEE Transactions on Vehicular Technology, vol. 66, pp. 5351-5363, 62017.

11. Y. Al-Nidawi, H. Yahya and A. H. Kemp, "Tackling Mobility in Low Latency Deterministic Multihop IEEE 802.15.4e Sensor Network," IEEE Sensors Journal, vol. 16, pp. 1412-1427, 32016.

12. Y. Al-Nidawi and A. H. Kemp, "Mobility Aware Framework for Timeslotted Channel Hopping IEEE 802.15.4e Sensor Networks," IEEE Sensors Journal, vol. 15, pp. 7112-7125, 122015.

13. A. Aijaz and U. Raza, "DeAMON: A Decentralized Adaptive Multi-Hop Scheduling Protocol for 6TiSCH Wireless Networks," IEEE Sensors Journal, vol. 17, pp. 6825-6836, 102017.

14. N. Accettura, E. Vogli, M. R. Palattella, L. A. Grieco, G. Boggia and M. Dohler, "Decentralized Traffic Aware Scheduling in 6TiSCH Networks: Design and Experimental Evaluation," IEEE Internet of Things Journal, vol. 2, pp. 455-470, 122015.

\section{AUTHOR PROFILE}

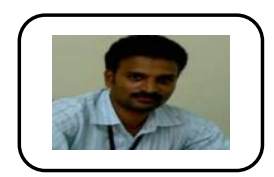

K. Suresh, Associate Professor, ECE, Chaitanya Engineering College, VSP, has research interests lie mostly in the field of IOT, Sensors, Wireless Communication Systems and System Integration.

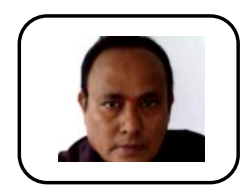

Dr. M. Ramesh Patnaik, Associate Professor, Department of Instrument technology, A.U. College of Engineering, Andhra University, is a fellow of the Institution of Engineers (India) and member of IETE and ISTE. He has quite a good number of publications is the field of fuzzy control, smart transmitters and general industrial control. His research interests lie mostly in the field of industrial control, fuzzy control, sensors and system integration

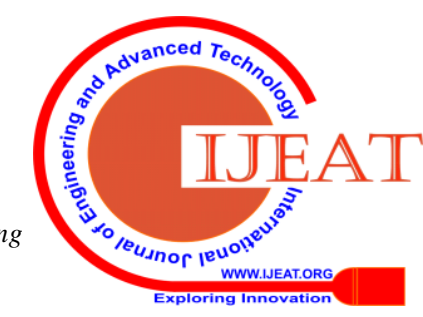

